\title{
Histochemical study of the oesophagus in the chukar partridge (Alectoris chukar) embryo
}

\author{
B. Shojaei ${ }^{1}$, S. Hashemnia², R. Eslami Rad ${ }^{2}$ \\ ${ }^{1}$ Department of Veterinary Anatomy, Faculty of Veterinary Medicine, Shahid Bahonar University of Kerman, Kerman, Iran \\ ${ }^{2}$ Department of Veterinary Histology, Faculty of Veterinary Medicine, Shahid Bahonar University of Kerman, Kerman, Iran
}

[Received: 31 October 2015; Accepted: 7 January 2016]

\begin{abstract}
Study of the histogenesis of different organs is a useful laboratory method which helps us achieve accurate basic information about organ development during the embryonic period. It also reveals histological differences of each organ in different species. This research was carried out to study the histogenesis of the oesophagus of chukar partridge. For this purpose, the embryonated eggs were placed in the incubator and the embryos were collected between the $5^{\text {th }}$ to $24^{\text {th }}$ days of incubation period. The specimens were fixed in Bouin's solution, and routine histotechnique processes were performed. The slides were finally stained with haematoxylin-eosin, Periodic Acid Schiff, Alcian Blue and Masson Trichrome, and the developmental changes of the oesophagus during the embryonic period were studied by light microscope. The four layers of oesophagus, including, the mucosa, submucosa, tunica muscularis and advantitia/serosa, both in cervical and thoracic oesophagus, were studied. During days 5 to 24 of incubation, developmental events in the oesophagus such as changes in the oesophageal epithelium, formation of muscularis mucosae and tunica muscularis, development of the mucous glands and the type of their secretion, were observed. Finally the results were compared with those of other studied avian species and the similarities and differences were discussed. (Folia Morphol 2016; 75, 4: 474-480)
\end{abstract}

Key words: Aves, gastrointestinal, histology, development, embryology

\section{INTRODUCTION}

The oesophagus as a passage between pharynx and stomach leads the masticated food to the lower parts of the digestive tract for further digestion. In the mammals, it is a muscular tube modified for voluntary and involuntary movement of foodstuffs to and from the stomach [2]. The oesophagus of birds shows histologic differences with that of mammals including glandular lamina propria [1, 2], different extension of muscularis mucosae into the mucosal folds $[5,9,11]$, absence of muscularis mucosa in some birds $[5,10]$ and two external smooth muscular layers covered almost completely by the adventitia $[1,2]$. Consequently, these differences result from different sequential events of the oesophagus histogenesis in these two classes of animals. Although the histogenesis of the oesophagus has been studied in some avian species [5, 13], differences in their nutritional behaviour, length of incubation period and evolutionary origin of avian species result in different time and sequence of the morphogenetic events of the oesophagus formation and have been prevented researchers to introduce a common "model of oesophagus hitogenesis" for them. 
The chukar partridge (Alectoris chukar), as a member of phasianidae family [3], belongs to the same family as chicken and has 3-4 days longer embryonic period. It would be interesting that to what extend the time and sequence of morphogenetic events of oesophagus histogenesis is similar to that of the chick embryo. The chukar partridge has also become popular in the Iranian food industry in recent years. Regarding the progressive interest of its meat and large investments in this field, providing knowledge of the microscopic anatomy and biology of this species could be quite valuable. Also, research on partridges in Iran has started very recently [8], which makes this study even more important as it is intended to be a reference for future studies. The present work was conducted to describe normal histological and histochemical findings on the histogenesis of the oesophagus of chukar partridge.

\section{MATERIALS AND METHODS}

Embryonated partridge (Alectoris Chukar) eggs were incubated at $37.5 \pm 0.2{ }^{\circ} \mathrm{C}$ with $56 \%$ humidity. From day 5, at least 3 embryos were studied, every $24 \mathrm{~h}$. First the eggs were checked for the embryos vitality by opening a window in the egg shell and direct observation of the blood circulation and/or embryo movement. The examined embryos were killed by freezing at $-20^{\circ} \mathrm{C}$ (5-14 days old) or decapitation (15-24 days old) and fixed in Bouin's solution.

The oesophagus of the embryos at least 3 specimens per day, was used for tissue processing from day 5 to 24. Paraffin blocks were prepared from the cervical and thoracic oesophagus and $5 \mu \mathrm{m}$ thick sections were obtained with a MR2258 microtome (HistoLine, Pantigliate, Italy). The slides were stained with haematoxylin-eosin* (H\&E), Periodic Acid Schiff* (PAS), Masson Trichrome* (MT) and Alcian Blue* (AB) methods and studied by light microscope. (*Merck, KGaA, 64271, Darmstadt, Germany).

\section{RESULTS}

Day $\mathbf{5}$ of incubation. The oesophagus was seen under the notochord and dorsal aortae and above the trachea. A common homogenous mesenchymal tissue surrounded both the trachea and oesophagus with no distinct boundary. The oesophageal lumen was very small and lined with pseudostratified columnar epithelium with some cilia (Fig. 1).

Day 6 of incubation. The surrounding mesenchymal tissue of the oesophagus divided into 2 layers: a com-

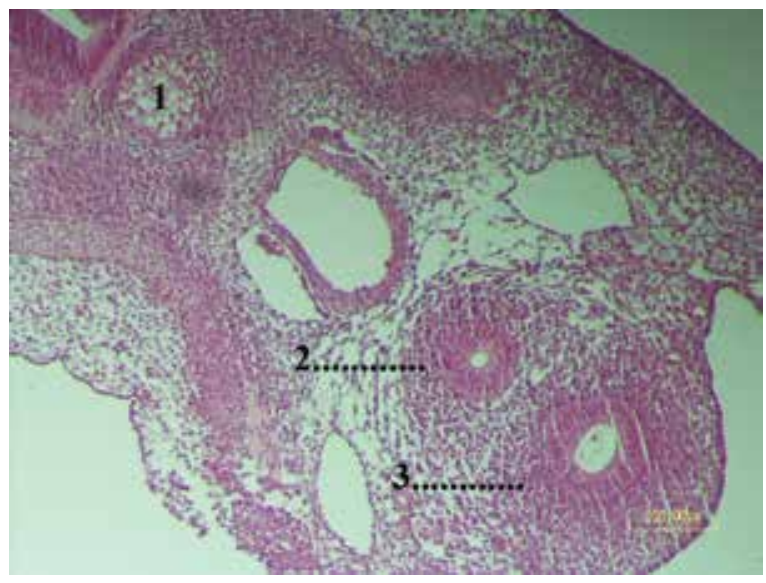

Figure 1. Oesophagus of the $5^{\text {th }}$ day of incubation; H\&E; 1 - notochord; 2 - oesophagus; 3 - trachea.

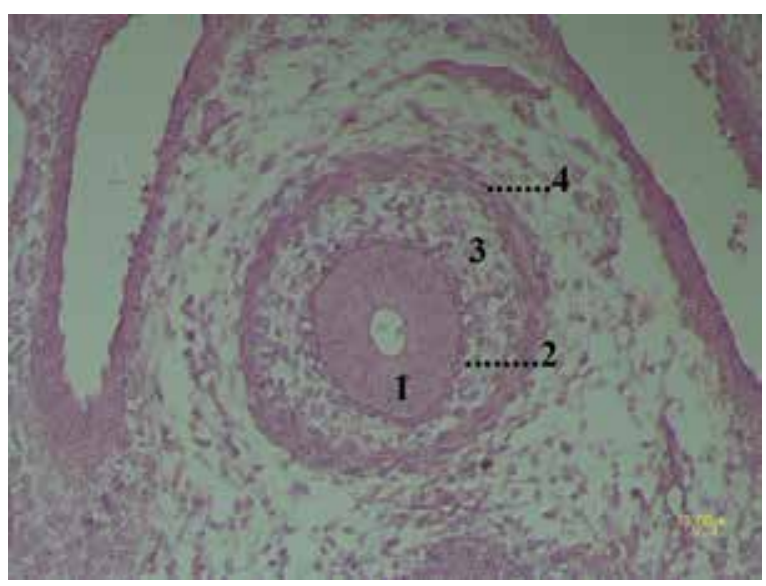

Figure 2. Oesophagus of the $7^{\text {th }}$ day of incubation; PAS; 1 - ciliated pseudostratified columnar epithelium; 2 - dense mesenchymal tissue; 3 - less dense mesenchymal tissue; 4 - inner circular layer of tunica muscularis.

pact inner layer just beneath the epithelium and a less dense outer layer. The former would be developed to lamina propria and the latter to submucosa. The lumen of the oesophagus appeared bigger than previous day and some acidophilic material could be seen in it. The epithelial tissue was ciliated pseudostratified columnar.

Day 7 of incubation. The outermost limit of the oesophagus at this stage was the band of developing circular muscle fibres of the tunica muscularis (Fig. 2).

Day 9 of incubation. The oesophagus was moving to the right of the trachea and under the skin. The epithelium changed into a 2 layered cuboidal tissue with some cilia. $A B$ positive secretions appeared in the lumen at the surface of the epithelial cells. No PAS positive secretion was observed (Fig. 3). 


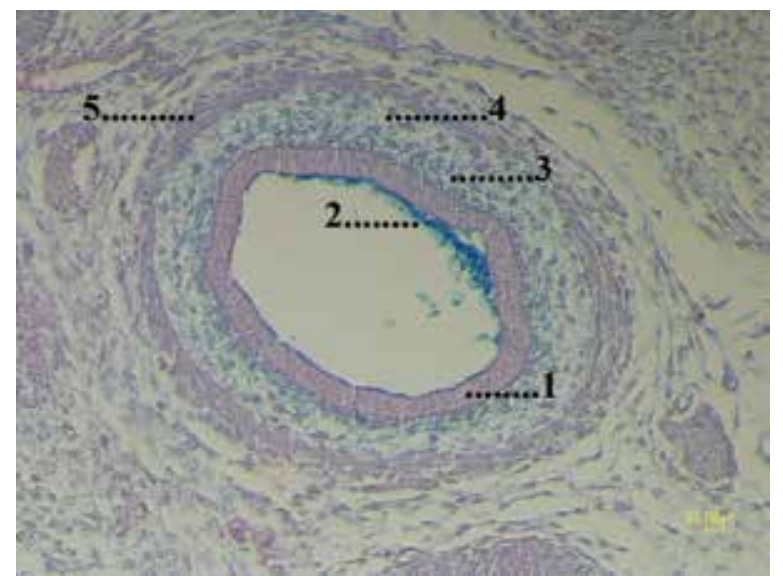

Figure 3. Oesophagus of the $9^{\text {th }}$ day of incubation; $A B ; 1-$ two layered cuboidal epithelium; $2-A B$ positive secretions; $3-$ dense mesenchymal tissue; 4 - less dense mesenchymal tissue; 5 - inner circular layer of tunica muscularis.

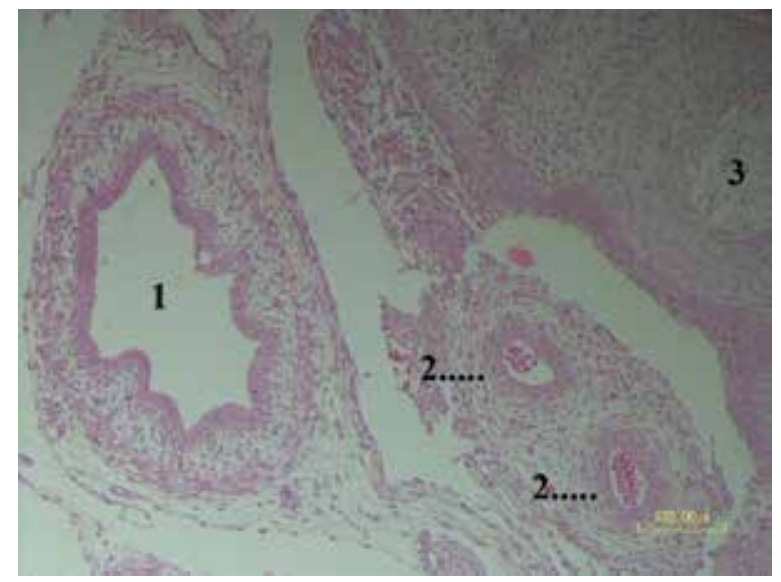

Figure 4. Oesophagus of the $11^{\text {th }}$ day of incubation; H\&E; $1-$ oesophagus with star shaped lumen; 2 - dorsal aorta; 3 - notochord.

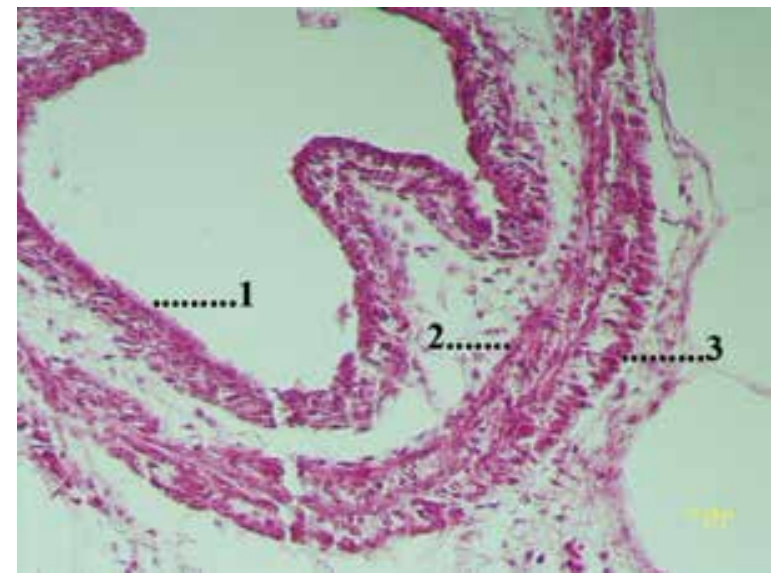

Figure 5. Cervical part of oesophagus of the $13^{\text {th }}$ day of incubation; $\mathrm{H} \& \mathrm{E} ; 1$ - cuboidal epithelium with numerous cilia; 2 - inner circular muscle; 3 - outer longitudinal muscle.

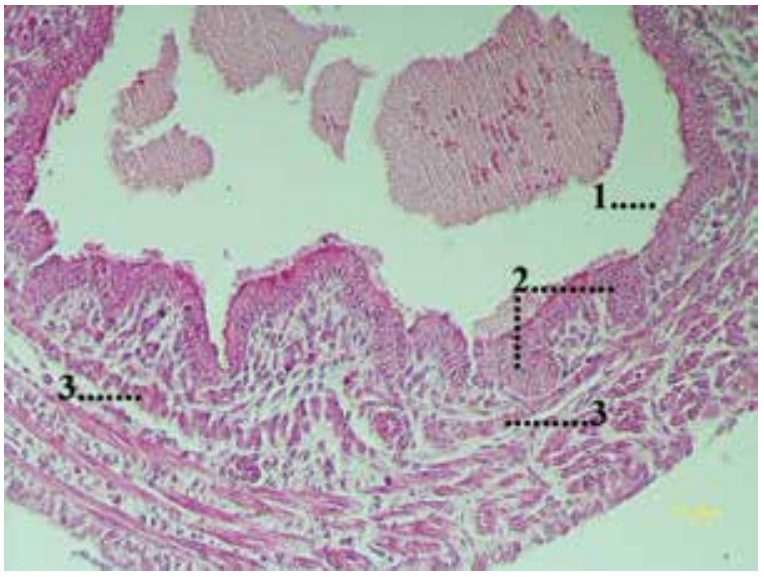

Figure 6. Thoracic part of oesophagus of the $15^{\text {th }}$ day of incubation; H\&E; 1 - cilia; 2 - epithelial buds; 3 - muscularis mucosae.

Day 10 of incubation. The oesophagus was completely positioned to the right side of the trachea. Its lumen was larger with the same epithelial tissue as day before, but more cilia were seen. The luminal secretions positively reacted to the techniques used in the present study, the PAS and AB stains. It can be concluded that they contained both neutral and acid GlycosAminoGlycans (GAG's).

Day 11 of incubation. The oesophagus had a star shaped lumen. An increase in the amount of cilia could be seen (Fig. 4).

Day 12 of incubation. Small blood vessels and capillaries were seen in the submucosa and lamina propria. Some ganglia of Auerbach's plexus were visible around the inner circular muscle.

Day 13 of incubation. The muscularis mucosa was not identifiable yet, but the outer longitudinal muscle appeared at this day (Fig. 5).

Day 14 of incubation. From this day until the end of the incubation period, the thoracic part of oesophagus speeded up the developmental process in comparison with the cervical part. The muscularis mucosae appeared for the first time only in the thoracic part at this day.

Day 15 of incubation. Solid epithelial buds, the forerunners of mucous glands, were formed in the lamina propria of the thoracic part. The muscularis mucosae and some ganglia of Meissner's plexus in the submucosa also were seen, while in the cervical part, no important change was visible (Fig. 6).

Day 17 of incubation. The muscularis mucosa was identified at this stage in the cervical part. In the thoracic part, the epithelial buds formed the mucous 


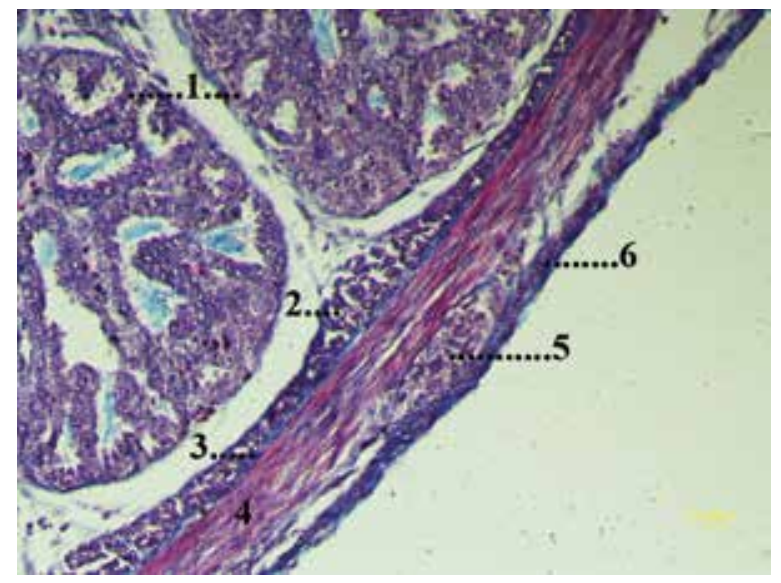

Figure 7. Thoracic part of oesophagus of the $18^{\text {th }}$ day of incubation; MT; 1 - well-developed mucous glands; 2 - muscularis mucosae; 3 - submucosa; 4 - inner circular muscle; 5 Auerbach's plexus; 6 - outer longitudinal muscle.

glands as their lumen appeared by vacuolation. Thick continuous muscularis mucosae, inner circular and outer longitudinal layer of tunica muscularis were seen more developed. Red and blue coloured secretions were present on the luminal surface of the oesophagus, in reaction to the PAS and $A B$ stains, respectively.

Day 18 of incubation. Two types of simple and pseudostratified columnar epithelial tissue were seen in the thoracic part of oesophagus. According to the large amount of secretions, detailed observation of the surface cells was not possible, so that the cilia were seen hardly. The mucous glands of the thoracic part had developed and started the secretion activity. Both the glands and the epithelial cells of the lumen had PAS positive secretion but the AB positive reaction was most seen at the luminal surface and less in the glands. The mucous glands were seen between the epithelium and the muscularis mucosae. They pushed the latter toward the tunica muscularis. The muscularis mucosa was best seen under the space between the two mucous glands. The thickness of submucosa was reduced greatly during the last days of incubation. So the muscularis mucosae appeared to be a part of tunica muscularis. In the MT stain, a very thin blue layer showed the location of submucosa. The tunica muscularis was also thin, especially the outer longitudinal layer which was discontinuous in some places (Fig. 7).

Day 19 of incubation. At this day, solid epithelial buds appeared in the lamina propria of the cervical part of oesophagus with great delay comparing with the thoracic part. The mucous glands of the thoracic part completely developed and occupied the most thickness of the wall. They were lined by simple cuboidal epithelium. Their excretory ducts were identifiable with simple columnar or cuboidal epithelial tissue in the epithelium. The acid and neutral GAG's secretion of glands increased compared with previous day, but the secretory activity of the luminal epithelium was still visible.

Day 20 of incubation. The epithelial tissue of the cervical part of oesophagus was stratified cuboidal and also stratified squamous in some parts and partially ciliated. Mucous glands were seen in two forms: solid epithelial buds and the ones with lumen. They were not active yet and the PAS and AB positive secretions seen only on the luminal surface. Some ganglia of Meissner's plexus in the submucosa were also observed. In the thoracic part of oesophagus nothing especial happened and only an increase of the $A B$ positive secretions in the glands were visible.

Day 21 of incubation. In the most area of the cervical part of oesophagus, the epithelial tissue became stratified squamous and the cilia decreased. Mucous glands formed as their lumen developed, and completely opened. They were lined by a simple cuboidal epithelium, which were still inactive. The compensatory secretions of acid and neutral GAG's were seen in the luminal surface. The thoracic part of oesophagus reached the adult histological pattern with well-developed large and functional mucous glands and well defined muscularis mucosae and tunica muscularis. The secretion of the glands increased but the luminal cells still had a small amount of both $A B$ and PAS positive secretions (Fig. 8).

Day 22 of incubation. The epithelial tissue of cervical part was a thin stratified squamous epithelium. The muscularis mucosa became thicker and was visible in the mucosal folds and base of the glands. The submucosa and the tunica muscularis with a thick inner circular and outer longitudinal layer were seen. The thoracic part was the same as the day before.

Day 24 of incubation. The epithelial tissue of the cervical part of oesophagus was a thin stratified squamous and some vesicles were seen in the epithelium. The cilia decreased and were limited to a few cells. Some of the mucous glands appeared to be functional, as their nucleus migrated to the base of the cell and the apical cytoplasm was visible. In the $A B$ and PAS stains, acid and neutral mucopolysacharides (GAG's) were detected both in the glands and on the 


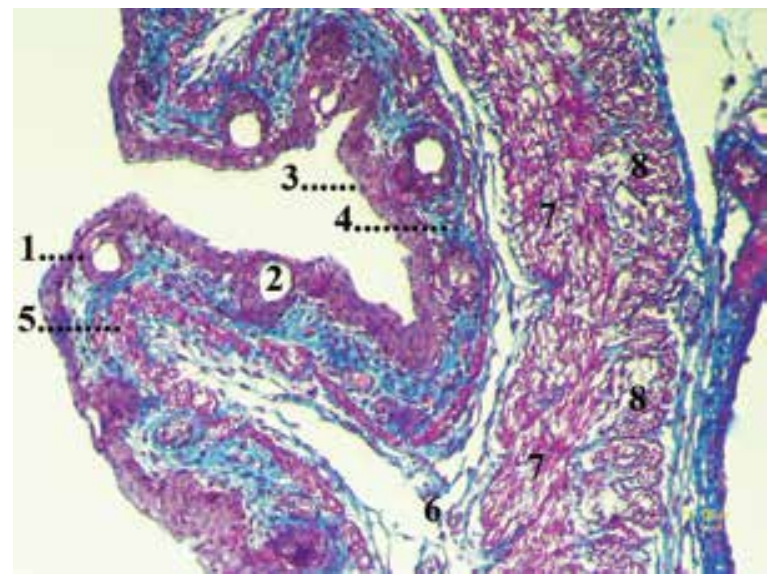

Figure 8. Cervical part of oesophagus of the $21^{\text {st }}$ day of incubation; MT; 1 - the epithelium of the glands; 2 - lumen of the glands; 3 - stratified squamous epithelium; 4 - lamina propria; 5 muscularis mucosae; 6 - submucosa; 7 - inner circular muscle; 8 - outer longitudinal muscle.

epithelial surface. The thoracic part was the same as the day before (Fig. 9).

\section{DISCUSSION}

One of the earliest researches on the histogenesis of the oesophagus is the study of epithelial tissue development of pig oesophagus in 1905 [14]. Five years later the histogenesis of human mucosa of oesophagus, stomach and small intestine was studied [6]. The avian oesophagus had also been the subject of this kind of studies, which most of them focused on the chicken embryo. Turkey, guinea fowl, pigeon and a few other birds species were also studied [4, 5, 13].

The present work was carried out to investigate the histogenesis of the oesophagus of chukar partridge (Alectoris chukar) and describe the development and disappearance of embryonic characters according to the incubation period which may indicate the degree of maturation before hatch.

In this study, the oesophagus of chukar partridge appeared as a compact area with a very narrow lumen at day 5 of incubation. The epithelial tissue of chukar partridge oesophagus was seen pseudostratified columnar on $5^{\text {th }}, 6^{\text {th }}$ and $7^{\text {th }}$ days of incubation. Then, from day 9, two types of epithelium were visible, simple cuboidal in small area and 2-layered cuboidal in most places. In the cervical part of oesophagus, this cuboidal tissue turned into stratified cuboidal first and finally converted to a thin stratified squamous epithelium on day 22. In the thoracic part, following the stratified cuboidal epithelium, a simple and pseudostratified columnar epithelium was seen until the

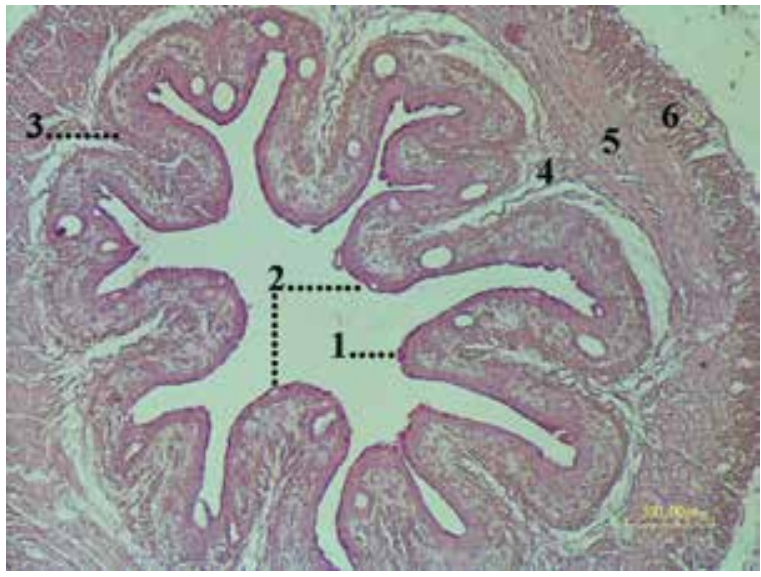

Figure 9. Cervical part of oesophagus of the $24^{\text {th }}$ day of incubation; PAS; 1 - the PAS positive secretion of the lumen; 2 - vesicles in the epithelium; 3 - muscularis mucosae; 4 - submucosa; 5 inner circular muscle; 6 - outer longitudinal muscle.

end of incubation period. Schumacher [12] described the oesophagus of the fowl as having a pseudostratified epithelium at $5^{\text {th }}$ day, low columnar at $7^{\text {th }}$ day, 2-layered cuboidal at $9^{\text {th }}$ day, and 4-layered stratified squamous at $18^{\text {th }}$ day. Ivey and Edgar [5] reported that the oesophageal epithelium of the chicken, turkey, guinea fowl and pigeon was simple columnar or rarely pseudostratified type in the youngest embryos. They described the elder embryos oesophagus with 2-layered cuboidal epithelium and sometimes, the surface layer stretched into the squamous type. There was a gradual increase in the number of cell layers, with the surface layer assuming a squamous form in some cases and a columnar or cuboidal type in others. The squamous type of surface cell became more common in later stages [5].

The oesophageal epithelial tissue of the chukar partridge embryo was not keratinized in the whole incubation period. On the contrary, Ivey and Edgar [5] reported the epithelium appeared to be somewhat horny in the pharynx, oesophagus, and crop of the 20-day chick embryo. However, in 2013, Ventura et al. [13] stated that the mucosa of the chicken oesophagus in 12- to 20-day-old embryos was covered by non-keratinised and secreting squamous stratified epithelium.

The cilia were also seen on the oesophageal epithelium of the chukar partridge embryo. They were easily visible on the pseudostratified columnar epithelium at the beginning and later on the simple, 2-layered and stratified cuboidal epithelium. On the day 20 of incubation, in the cervical part of oesopha- 
gus, there was a decrease in the amount of cilia, which were partially seen. After appearance of the stratified squamous epithelium, the cilia were rarely visible and were limited to small islands of ciliated cells. In the thoracic part, the cilia were observed on the columnar cells until the end of incubation period. Schumacher [12] studied the chick embryo oesophagus and although he searched for ciliated cells, he failed to observe them. The investigation of Ivey and Edgar [5] showed that in the turkey, ciliated cells were seen first in the 16-day specimen. From the $20^{\text {th }}$ day to hatching, ciliation ranged from $50 \%$ to $80 \%$ in the cervical part. In the thoracic part ciliation decreased from $90 \%$ at $23^{\text {rd }}$ day to $40 \%$ at hatching. In the chick, cilia appeared first in the thoracic part of the 13-day embryo, with about $80 \%$ of the surface being covered with ciliated cells. In the 16-day specimen, cilia were present on approximately $10 \%$ of the epithelium in the thoracic part. In the guinea fowl, cilia were seen only in the thoracic part of oesophagus and no cilia were visible in the oesophagus of the pigeon [5]. Comparing these results with those of chukar partridge, it was totally different with the guinea fowl and pigeon but there was some similarity in the presence of cilia in both cervical and thoracic oesophagus with the turkey and chick embryo although the time of their appearance was much later than that of the chukar partridge embryo.

The mucous glands in the avian oesophagus first appear as epithelial buds during the second half of incubation and their lumens form by intercellular vacuolation [12]. In the present study, the solid epithelial buds were appeared first in the thoracic part of oesophagus in $15^{\text {th }}$ day of incubation. They developed very fast and started their secretion activity by day 18 . In the cervical part, the epithelial buds appeared much later at $19^{\text {th }}$ day of incubation. They had a very slow development as the glands formed on day 21 and still inactive. Their epithelium was simple cuboidal. At the end of the incubation period, day 24 , some of the mucous glands of the cervical part seemed to be functional and had some secretions. The secretory activity in the cells of the glands was indicated by the presence of apical cytoplasm and movement of nuclei toward the base of the cells and in the epithelium it was indicated by the presence of vesicles which sometimes projected into the lumen as the result of accumulation of the secretions. They were continuous with the lumen of mucous glands. Ivey and Edgar [5] reported that epithelial buds were seen first in the thoracic part of oesophagus of the 13-day chick and 16-day turkey, in the oesophageal portion of the crop and thoracic part of oesophagus of the 16-day guinea fowl, and in the crop of the 13-day pigeon. In the 20-day turkey, buds were present throughout the oesophagus and crop, but glands with lumen were present only in the thoracic part [5]. Ventura et al. [13] stated that the primordia of oesophageal glands of the chick embryo were already present on the $12^{\text {th }}$ day of development. From the $16^{\text {th }}$ day on, the cells that formed the oesophageal glands organised themselves in 1 or 2 layers around the lumen. Only on the $20^{\text {th }}$ day the cells of the glands became characteristic of mucous glands: tall and with nucleus located at the basal region. It is not clear that these results belong to the cervical or thoracic part of the oesophagus as they had not mentioned it in their study [13].

In the present study, the type, location and the time of GAG's secretions were identified by using $A B$ and PAS stains. $A B$ positive secretions first appeared in the lumen at the surface of the epithelial cells on the $9^{\text {th }}$ day of incubation. From day 10 the luminal secretions were PAS positive and the secretion of both neutral and acid GAG's continued until the end of incubation period. We detected the mucous glands secretion in the thoracic part much earlier than the cervical one. Both $A B$ and PAS positive secretions of the glands were seen on day 18 in the thoracic part of oesophagus and shortly before hatching in the cervical part. In the investigation by Ventura et al. [13] on the chick embryo, the cells of the luminal epithelium produced both types of GAG's, neutral and acid, from the $12^{\text {th }}$ day to the $18^{\text {th }}$ day which indicated that in this stage the epithelium was secretory. In their research the oesophageal glands were not active until the $16^{\text {th }}$ day, weakly positive from the $17^{\text {th }}$ to the $19^{\text {th }}$ and strongly positive on the $20^{\text {th }}$ day [13].

At first the lamina propria and the submucosa were a non-distinguishable mesenchymal tissue. On the day $6^{\text {th }}$ of incubation, the lamina propria appeared as a layer next to the epithelium that was usually more compact than the rest of the mesenchyme and could be distinguished from the submucosa even before the muscularis mucosae was formed. According to the Ivey and Edgar study [5], the mesenchyme in the turkey and pigeon was not as dense as in comparable stages of chicken and guinea fowl.

In the histogenesis of the smooth muscle layers of the chukar partridge oesophagus, the circular layer 
of the tunica muscularis was the first to be formed then the longitudinal layer and lastly, the muscularis mucosae. The inner circular and the outer longitudinal layers appeared on the day 7 and 13 respectively, but the formation of the muscularis mucosae was different in the two parts of the oesophagus as it was developed 3 days earlier in the thoracic part than the cervical one.

Kendall [7] claimed that in the pigeon the muscle layers developed in an unusual manner. The longitudinal layer of the tunica muscularis was either poorly developed or absent, and the submucosa was reduced greatly during the last quarter of incubation. Thus, the muscularis mucosae appeared to be a part of the tunica muscularis. The adult pigeon oesophagus has been described as having no muscularis mucosae but having an inner longitudinal layer and an outer circular layer [7]. These results in the pigeon seems to be similar to the chukar partridge in this study, because during the last days of incubation, the submucosa of the thoracic part of oesophagus was very thin and seemed to be absent in some places, but the MT stain, revealed it as a thin blue line between the muscularis mucosae and the inner circular layer. The outer longitudinal layer was thick in the cervical part and very thin and discontinuous in thoracic part.

\section{CONCLUSIONS}

If we assume a similar pattern of oesophagus histogenesis for chukar partridge and chicken, we expect the developmental events take place by similar sequences and intervals in these two birds. In the cervical part, the stratified squamous epithelium was first seen in the $18^{\text {th }}$ day and $22^{\text {nd }}$ day in the chicken and chukar partridge, respectively, which may be due to shorter incubation period of the chicken compared with the chukar partridge. If so, we would expect to see other developmental events 3-4 days earlier in the chick embryo as it happens for epithelial buds in the thoracic part. But this interval is not observed in all oesophageal developmental events such as the conversion of the epithelial tissue or the onset of secretion of the mucous glands. Furthermore, the appearance of the cilia in oesophagus of chukar partridge was much earlier comparing with other studied species. They were seen from the first day of study, until the end of incubation period. The results of the present study indicate that the oesophageal developmental events in the chukar partridge follow different intervals which are not compatible to the difference in incubation period of these two birds.

\section{Acknowledgements}

This research was financially supported by the Research Council of Shahid Bahonar University of Kerman.

\section{REFERENCES}

1. Bacha WJ, Bacha LM (2012) Color atlas of veterinary histology. $3^{\text {rd }}$ Ed. ISBN-13: 978-0470958513.

2. Banks WL (1993) Applied veterinary histology. $3^{\text {rd }} \mathrm{Ed}$. Mosby, USA, pp. 336-337.

3. Del Hoyo J, Elliott A, Sargatal J, Christie D (1992) Handbook of the birds of the world. Vol. 2. New World Vultures to Guineafowl. ISBN 84-87334-20-2.

4. Edgar SA (1947) Ciliated epithelial cells lining the oesophagus of young turkey and chick embryos. Poultry Sci, 46: 525-534.

5. Ivey WD, Edgar SA (1952) The histogenesis of the esophagus and crop of the chicken, Turkey, Guinea fowl and pigeon with special reference to ciliated epithelium. Anat Rec, 114: 189-211.

6. Johnso NP (1910) Development of the mucous membrane of the esophagus, stomach and small intestine in the human embryo. Am J Anat, 10: 521-575.

7. Kendall JI (1947) The microscopic anatomy of vertebrates. $3^{\text {rd }}$ Ed. Lea and Febiger, Philadelphia.

8. Mobini B (2012) Microscopic study of the gall bladder of the chukar partridge (Alectoris Chukar). BJVM, 15: 73-78.

9. Parchami A, Dehkordi RAF (2011) Histological characteristics of the esophageal wall of the common quail (Coturnixcoturnix). World Appl Sci J, 14: 414-419.

10. Rossi JR, Baraldi-Artoni SM, Oliveira D, da Cruz C, Sagula A, Pacheco MR, de Araujo ML (2006) Morphology of oesophagus and crop of the partridge Rhynchotus rufescens (tiramidae). Acta Sci Biol Sci, 28: 165-168.

11. Sagsoz H, Liman N (2009) Structure of the esophagus and morphometric, Histochemical- Immunohistochemical profiles of the oesophagus gland during the post hatching period of Japanese quails (Coturnixcoturnix japonica). Anat Histol Embryol, 38: 330-340.

12. Schumacher S (1926) Die Entwieklung der Glandulae oesophageae des Huhnes. Nebst Bemerkungen uber die Bildung der Drusenlichtung im allgemeinen and uber den Epithelumbau im Osophagus des Huhnes. Z Mikrosk Anat Forsc, 5: 1-22.

13. Ventura $A$, do Nascimento $A A$, dos Santos MAJ, VieiraLopes DA, Sales A, Pinheiro NL (2013) Histological description of morphogenesis of the gastroesophageal mucosa of Gallus Gallus Domesticus. (Linnaeus 1758). Int J Morphol, 31: 1331-1339.

14. Whitehead RH (1905) A note on the development of the esophageal epithelium. Am J Anat, 4: 6-7. 